\title{
The Practice of Geriatrics: Specialized Geriatric Programs and Home Visits
}

\author{
David B. Hogan, MD, FRCPC, FACP \\ Professor and Brenda Strafford Foundation Chair in Geriatric Medicine, University of Calgary, Calgary, AB
}

\begin{abstract}
Home visits have a long history in geriatrics. In this narrative review, the literature on home visits performed by specialists in geriatric medicine (or psychiatry) and/or specialized programs in geriatric medicine (or psychiatry) published between January 1988 and December 2008 was examined. The papers reviewed were few and inconsistent in their message. The lessons that can be derived from them are limited. Draft recommendations about the role of home visiting by specialized geriatric programs in Canada are presented.
\end{abstract}

Keywords: home visits, house calls, narrative review, specialized geriatric programs

\section{INTRODUCTION}

House calls (also known as home or domiciliary visits) are visits by physicians to examine patients in their own homes. They have a long history in the care of older patients. Nascher, who coined the word geriatrics, made frequent house calls to his patients. He grew to know New York so well he edited a guidebook to the city. ${ }^{1}$

In the U.K., Eric B. Brooke is credited with introducing the practice of domiciliary visits by consultants in geriatrics during the early years of the National Health Service (NHS). ${ }^{2}$ After the Second World War, when faced by increasing numbers of older people seeking admission, a finite and inadequate number of beds, and pressure to do something about this mismatch, Brooke began visiting those waiting in their homes. He found that many didn't require a hospital admission to sort out their problems. His wait list was pruned while the care of older individuals in his district improved. His visits were described as follows:

With Dr. Brooke was his registrar and his secretary. First he had a short talk with the relatives; then he investigated the patient. Then the relatives, the secretary and Dr. Brooke (or the registrar) went over the whole matter and gave advice and decisions, and the secretary made the necessary notes for the case-record. It was all simple, easy, free offuss. The procedure had a wonderful effect on waiting-lists, and Dr. Brooke was, through his secretary, in constant friendly touch with doctors. ${ }^{3}$
During the middle years of the last century, older patients in the U.K. were routinely seen in their homes before admission to a geriatric unit. Arcand and Williamson described this practice in a Scottish professorial unit near the end of its heyday. ${ }^{4}$ An astounding $89 \%$ of patients were seen at home on the day of referral with two-thirds within 3 hours of the request. Forty-one percent of those seen were subsequently admitted ( $78 \%$ to a geriatric bed). The paper by Brooke referenced by Arcand as justification for home visits recommended that they be performed by social workers, not physicians, and expanding ambulatory services. ${ }^{5}$ Lord Amulree and colleagues in a 1951 paper had made a stronger argument for home visits by consultants in geriatrics. ${ }^{6}$

Over the last quarter century the frequency of home visits in the U.K. has declined precipitously. In 1986 U.K. consultants in geriatric medicine performed an average of 187.3 domiciliary visits per year (about 3-4 per week). This dropped by $21.4 \%$ over the next 5 years. ${ }^{7}$ Between 1990 and 1993 , there was a $32 \%$ decrease in domiciliary visits by geriatricians in Northern Ireland. The need for them had become less pressing. General practitioners (GPs) were now able to arrange direct admissions to hospital for their older patients, refer them to urgent outpatient clinics, and/or obtain immediate access to geriatric day hospitals. ${ }^{8}$ Concurrently the number of NHS geriatric beds declined at approximately the same rate. Between 1990 and 1998 approximately a third of these beds were lost. ${ }^{9}$ Proportionally more older patients were admitted through the emergency department with fewer directly admitted from the community after a domiciliary consultation. A seminar of Scottish geriatricians reminiscing about domiciliary visits sheds light on the reasons for both the rise and then decline of home visits - and what may have been lost as a consequence. ${ }^{10}$

Even more rapid changes occurred in North America. ${ }^{11}$ In 1930 an estimated $40 \%$ of physician-patient encounters occurred in the patient's home. By 1993 only $0.88 \%$ of older American Medicare patients received a house call from a physician. ${ }^{12}$

A wealth of information can be obtained during a home visit otherwise not available. But is this always necessary for the care of patients? If it is, can it be obtained in a more efficient way? Criticisms of home visits include the belief that they are an inefficient use of limited physician time, the difficulty of doing a proper physical examination and/

(C) 2011 Author(s). Published by the Canadian Geriatrics Society. This is an Open Access article distributed under the terms of the Creative Commons Attribution Non-Commercial No-Derivative license (http://creativecommons.org/licenses/by-nc-nd/2.5/ca/), which permits unrestricted non-commercial use and distribution, provided the original work is properly cited. 
or obtaining investigations in the home, and concerns about practitioner safety. The latter is not a trivial issue. A British study found that $62.5 \%$ of all incidents of injurious assaults of GPs occurred during home visits. ${ }^{13}$ The Australian Medical Association has published guidelines for the protection of physicians undertaking home visits. Recommendations include providing escorts upon request, keeping timetables for scheduled home visits, reporting in at the end of each visit, following predetermined procedures if a physician can't be contacted or did not check in when expected, and ensuring that physicians carry a duress alarm and/or mobile phone (GPS-linked if necessary) during visits.

In this paper the relevant literature on home visits will be reviewed with provisional recommendations made on their appropriate role within specialized geriatric programs.

\section{METHODS}

The English-language literature on home visits performed by specialists in geriatric medicine (or psychiatry) and/ or specialized geriatric (or geriatric psychiatry) programs published between January 1988 and December 2008 was reviewed. Medline searches restricted to the English language and age 65+ were done using "home visit(s)," "house call(s)," and "domiciliary visit(s)" as search terms. The titles and abstracts of identified papers were reviewed. Based on this, some were selected for detailed study. The references of these articles were in turn searched for additional papers. The emphasis was on home visits performed at the request of primary care physicians to assess common geriatric syndromes like confusion and falls. This review doesn't deal with the primary medical care of older patients, palliative home care, hospital-at-home programs, home care services, or preventive home visiting.

\section{RESULTS}

The available literature is relatively sparse and methodologically weak. There are no randomized controlled trials with blinded assessment of outcomes. The lessons that can be derived from it are limited. Some of the selected articles are becoming dated. The frequency of home visiting declined markedly during the time period examined.

\section{Home Visits by Specialists}

Most papers dealing with this topic come from the U.K. The unique history, structure, and funding of health care in the U.K. makes it difficult to extrapolate their findings to other countries. Within the NHS, GPs can ask consultants to see patients in their homes for advice on diagnosis and treatment if these patients are unable to come to an outpatient visit because of their medical condition. An expectation is that the GP will be present during the visit. During the time-frame of this review domiciliary visiting was one of the few clinical services in the NHS that provided an extra fee to the consultant.

Mulley, in a paper about home visiting by consultants, stated that they were being used for purposes other than advising GPs. ${ }^{14}$ They were also being done to deal with urgent consultation requests quickly and to assess the appropriateness of a patient for a hospital admission. Most home visits were to older people with psychiatric or nonspecific symptoms. He noted that "...the charge that some visits are unnecessary cannot be ignored." Potential advantages for them (compared to an outpatient assessment) suggested by the author included the ability to obtain collateral history from people who tend not to come in to clinic visits; more valid cognitive evaluation; ability to look for signs of self-neglect (e.g., aroma of urine); ability to inspect the home for medications, fresh food, signs of alcohol abuse, bed not slept in, problems with hygiene, damp, poor lighting or heat, accident hazards, and environmental hindrances; ability to assess caregivers; chance to reassure the patient; ability to obtain blood samples and/or an ECG; teaching opportunities; and, after a home visit, improved ability to develop a realistic management plan. Potential disadvantages were that physical examination is often difficult; making home visits is time consuming; home visits are expensive; and it is unclear whether they are cost effective.

Hardy-Thompson and colleagues found that most (67\%) GPs who completed a questionnaire indicated that a psychogeriatric domiciliary visiting service was very important to their work. ${ }^{15}$ Both psychogeriatric consultants who participated in the study felt that "a proper assessment of dementia [the commonest clinical indication for a visit] meant seeing the patient's home and an informant." The same authors compared high GP users of the psychogeriatric domiciliary visiting service (i.e., GPs who requested $2+$ domiciliary visits in the last 6 months) with less frequent users. ${ }^{16}$ High GP users were more satisfied with the service, were more likely to come to the visit, and felt that physical disability was an important factor in making their request. They were less likely to have psychiatric training and more likely to feel susceptible to pressure from the patient's family. Several years later one of the co-authors of these papers wrote that old-age psychiatrists "cannot...justify home visits on the emotional basis of our personal fondness for them...particularly...in light of the growing popularity of multidisciplinary community teams in old age psychiatry... [which] allow for such assessments to be carried out by any team member." ${ }^{17}$ An audit found that less than a third of domiciliary consultations by a psychiatric service met required criteria for their performance. ${ }^{18}$

Donaldson and Hill reported that most consultants and GPs in the U.K. used domiciliary consultations sparingly, with GPs rarely turning up for them. ${ }^{19}$ They raised several concerns: the problems being seen were often minor; at times visits were being done as a prerequisite for admission to hospital; and some those seen could have come in for an assessment. Peer-review auditing led to a substantial decline in the number done. An accompanying editorial concluded that 
home visits "do seem to have a place, albeit a limited one." 20 Reardon et al. retrospectively reviewed domiciliary consultations carried out by two hospital-based care-of-the-elderly physicians in the U.K. ${ }^{21}$ Over a year one physician did 184 consultations with the other performing 268. Respectively, $29.9 \%$ and $20.9 \%$ of their patients were admitted to hospital after consultations. The equivalent figures for geriatric dayhospital admissions were $23.9 \%$ and $29.1 \%$. The consultants felt they had "something to offer" in $92.4 \%$ and $81.3 \%$ of the consultations, respectively, concluding that domiciliary visits were a "useful service." Crome et al. conducted an audit of domiciliary consultations done over a 3-month period in their region of the U.K. ${ }^{22}$ Six geriatricians performed 234 consultations (range in the number of visits/physician was 0-74). Most (78.6\%) visits occurred after normal work hours. GPs rarely (1.6\%) attended. The most common reasons given by GPs for having their patients seen at home were either difficulty in attending an outpatient visit due to the severity of the patient's illness (31.9\%) or immobility (26.4\%). Rarely (4.6\%) was it because the GP felt that it would be better to have the patient assessed at home. The authors suspected the "real" reasons were to have patients seen more quickly or to expedite hospital admission. Nearly a quarter (24\%) of those seen were admitted. Most (93.5\%) GPs thought the visits were of value.

None of the studies reported objective patient outcomes. While there seems to be a role for home visits by specialists, it appears that many of those being performed in the U.K. were not absolutely required. As Mulley concluded, “....if they are to continue as an important part of our health service they must be done responsibly and evaluated thoroughly."14

\section{Cognitive impairment/dementia}

In the U.K. patients with dementia are often initially seen in their own homes by geriatric psychiatrists. There is pressure on these programs to reallocate resources from home visiting to memory clinics. A study compared 76 consecutive new referrals to a geriatric psychiatry service for assessment of memory problems where the patient was assessed in a memory clinic versus 74 consecutive new referrals to the same service where a specific request was made for a domiciliary visit to assess the patient's memory concerns. ${ }^{23}$ Both were done over the same period of time. The domiciliary group had more behavioral and psychological problems. The authors felt memory clinics could complement domiciliary services but not replace them.

Another study looked at 64 consecutive referrals to a memory clinic. ${ }^{24}$ A community psychiatric nurse initially assessed them at home using a semistructured interview. A nurse diagnosis of dementia agreed well with the final clinic diagnosis, but there was only moderate concordance on the type of dementia. The authors felt that a physical examination and neuroimaging were important in making a specific etiological diagnosis. An earlier study had come up with similar findings. ${ }^{25}$ While the high concordance in diagnosing dementia is reassuring, probable dementia can be detected using informant questionnaires. ${ }^{26} \mathrm{In}$ a research study identification of the presence of dementia by trained nurse assessors after a 90-120 minute in-home assessment showed very good agreement with the final consensus diagnosis $($ kappa $=0.84)$, and good agreement with regard to the type $(\mathrm{kappa}=0.71) \cdot{ }^{27} \mathrm{~A}$ specialist nurse role was developed in a U.K. memory clinic. ${ }^{28}$ These nurses conducted a home-based assessment, formulated an initial diagnosis, and facilitated further assessments/investigations. All patients were subsequently seen by another discipline with the final diagnosis made at a multidisciplinary meeting led by a consultant in old-age psychiatry. This retrospective 18-month study compared the diagnosis made by the nurse with the one made at the multidisciplinary meeting. The two nurses saw 184 and 220 patients, respectively, over the 18 months (127-147/ year). There was very good agreement on the diagnosis of dementia (kappa $=0.88)$ and good agreement for the type of dementia (kappa $=0.76)$. The authors concluded that "nurse screening" was a way to provide an earlier diagnosis. The multidisciplinary team review was felt to guard against inaccurate diagnoses and/or inappropriate care. It was concluded that there was an "unrealized potential for nurses to become more involved in diagnosing early and uncomplicated cases of dementia in the community."

An American study compared a structured clinic-based assessment of older demented patients with a similarly structured home assessment. ${ }^{29}$ The focus here was not on the diagnosis of dementia but on the identification of problems. Trained geriatric nurse specialists conducted the assessments. The ones done at home were taken as the "gold standard." A staggering 1,751 problems were identified ( 8.76 per patient) in the patients enrolled in this study, with many of the identified problems given a high-risk score (defined as risk of death or serious morbidity some time in the future) by the investigators. Many $(n=622)$ problems were identified only during the home visit, but a nearly equal number $(n=597)$ were only identified during the clinic visit. Most (61\%) of the home visits lasted $90+$ minutes compared with $12 \%$ of office visits. Longer visits led to the identification of more problems. While the authors concluded that home visits were beneficial, methodologically this was a weak study. About as many problems were identified only during clinic visits as on home visits. No data on patient outcomes were presented. An unanswered question was whether the identification of all these problems actually led to changes in management and better outcomes. These authors published a paper 15 years earlier with similar results and conclusions. ${ }^{30}$

The location of the evaluation can have an impact on the results obtained on even brief cognitive measures. A study of older patients in a family practice found that Mini-Mental State Examination scores were on average 0.5 points higher if done at home than in the office. Patients tended to do better on three-item recall and spatial orientation. ${ }^{31}$ In another 
study the average difference between the two settings was 1.5 points with differences of $5+$ points in $25 \%$ of cases. Most (76\%) participants did better at home. ${ }^{32}$ It isn't clear which score is more useful for either assessment or management, but this is another source of variability in the results obtained on testing that should be kept in mind.

Trained nurses who are part of a multidisciplinary service can diagnose dementia on home visits, but there is less confidence in their ability to make an accurate determination of the likely cause. Whether the additional information obtained during a home visit influences management and improves outcomes can't be answered by the available literature.

\section{Falls}

A quasirandomized controlled trial enrolled 95 women aged $60+$ living in the community receiving in-hospital rehabilitation for a fall-related hip fracture. ${ }^{33}$ The women were alternately allocated to the intervention or control group. All took part in a fall prevention program during their inpatient stay with the intervention group also receiving a home visit for an environmental assessment by an occupational therapist a median of 20 days after discharge. Falls within the first 6 months were examined. A total of 13/50 in the control group had 20 falls over 9,231 days of observation. In the intervention group $6 / 45$ had nine falls over 8,970 days. There was a significantly lower proportion of fallers in the intervention group (odds ratio $0.275,95 \%$ confidence internal $0.081-0.937$, $p=0.039)$. The authors concluded that an occupational therapist home visit after discharge significantly reduced the risk of falling in this study. Positive results were also found in an earlier study of high-risk (i.e., those reporting 1+ falls in the year prior to recruitment) patients being discharged from hospital. ${ }^{34}$

A systematic review concluded that home safety interventions were possibly effective in preventing falls if they were part of a multifactorial intervention, or targeted to those with severe visual impairment or otherwise at high fall risk. ${ }^{35}$

\section{Medications}

One reason commonly given for a home visit is to more accurately assess the medications being consumed. One study compared drug histories obtained by an internist in a clinic with those obtained by a nurse practitioner during a home visit. ${ }^{36}$ The two lists disagreed $32 \%$ of the time with roughly equal numbers of "extra" drugs noted in the two settings. It wasn't clear which one was more accurate. The authors argued "against uncritical acceptance" of the widely held assumption that the drug history obtained on a home visit was the gold standard. To accurately determine drug use the authors suggested asking repeatedly, having all medications brought to clinic visits, and getting data from additional sources (e.g., pharmacy records, administrative databases).
In a Canadian study, medications identified at an inhome assessment (by semistructured interview followed by a room-by-room search) were compared with lists obtained at clinic visits (by interviewing the patient and examining all their medications, which they were asked to bring). ${ }^{37} \mathrm{In}$ $23 / 48$ patients (48\%) the clinic list missed at least one regular medication ( $9 / 48$ a prescribed one). At the clinic visit, eight patients $(17 \%)$ reported taking a medication that was not found on the home visit. The in-home interviews/inspections took 42 minutes making them impractical for routine use. More explicit questioning at the time of the clinic visit coupled with clearer previsit directions to look for all medications in the home might improve the yield of lists obtained during a clinic visit.

\section{Miscellaneous}

In a Canadian study, Clarfield and Bergman described how medical services were arranged for a group of 105 housebound seniors in an urban setting. ${ }^{38}$ After being identified, they were assessed and stabilized by a specialized geriatric service before being matched to family physicians willing and able to assume their care. Of those followed at 1 year, most $(69 \%)$ were still at home. The majority $(83 \%)$ were pleased with their medical care. The authors concluded that assessment and stabilization coupled with backup consultative services made it possible to find family physicians willing to assume the care of these housebound patients. Most of these housebound patients were still at home a year later.

Home visits can play a role in the education of practitioners. During a family medicine clerkship in Israel, family physicians visited a bedridden older patient at home with a small group of medical students and a geriatrician from the local hospital. Students rated the home visit highly. The experience allowed students to learn aspects of geriatrics not previously presented. ${ }^{39}$

\section{RECOMMENDATIONS}

Within specialized geriatric programs, home visits have evolved from a service performed by physicians for a specific indication (screening for appropriateness of hospital admission) to one done by variety of professionals for diverse reasons. While assessing the need for hospitalization is still a valid reason for a home visit, most admissions to a geriatric unit now occur through emergency departments, through clinics, or as intra-/interhospital transfers.

Home visits remain an important component of the comprehensive suite of services required by frail seniors. It is an activity that can be ramped up quickly, as the required infrastructure is minimal. More rigorous research on home visits is needed. We must be better at defining who should be seen at home (and by whom); determining what specifically should be done; examining how best to link home visits with our other activities, primary care, and community-based 
programs; and evaluating their effectiveness. Safety issues coupled with the time and resource implications of these visits mean that they should be targeted and planned. The potential role of old (e.g., telephone $)^{40}$ and emerging technologies (e.g., home-based information and communication technologies) to augment or replace home visits should be examined. ${ }^{41}$ Telephones, for example, can be used to screen/triage, assess, monitor/follow, and counsel/reassure patients.

The American Geriatrics Society developed a clinical practice statement on house calls in geriatric practice. ${ }^{42}$ Based on this, the literature review, and personal experience, provisional recommendations about home visits for Canadian specialized geriatric programs are proposed as a starting point for further discussion (see Table 1).

They should be a planned service and not an afterthought. Decisions about who will perform the visit should be driven by patient need, available resources, program structure, and professional judgment about appropriateness. Particular challenges for physicians are determining which ones should be done personally and how best to develop and maintain skills in assessing and managing patients in their own homes.

TABLE 1.

Provisional recommendations about home visits for specialized geriatrics programs in Canada

Principles:

Specialized geriatric programs should

1. Make provisions for home visits to meet the needs of select patients (ACCESS);

2. Provide evidence-based care of a high standard (QUALITY OF CARE);

3. Make efficient use of their available resources (SUSTAINABILITY).

\section{Criteria for home visits:}

1. Home visits should be performed when necessary. Initial (first contact from the standpoint of the service) assessment home visit should be considered for patients who

a. Have

i. a severe mobility disability where coming to a clinic appointment would be either impossible or very difficult (i.e., pain/ discomfort, effort, cost, and/or logistics) from the standpoint of the patient or their caregivers to accomplish;

ii. a high risk for falls where an environmental assessment is required;

iii. severe and disruptive behavioral problems making a clinic visit problematic (note: the safety of the provider will have to be considered if a home visit is offered);

iv. end-stage terminal illness;

b. Have no access to transportation for a clinic visit

c. Refuse to come in for a clinic visit but are willing to be seen in their home.

2. Home visits may be required to expedite the assessment of a patient with urgent concerns to determine whether he or she requires admission to hospital.

3. Home visits may be required to complete the assessment of a patient already seen in either an ambulatory or inpatient setting. Reasons would include a need to directly assess the physical and social environment of the patient (e.g., look for safety issues, confirm a suspicion of neglect, abuse and/or caregiver burden), directly assess function in the person's own residence, obtain important collateral information that cannot be obtained otherwise, evaluate medication availability and consumption, and/ or develop a management plan with realistic goals.

4. Member(s) of a multidisciplinary team working in a specialized geriatric program may perform home visits as part of an integrated and/or staged assessment of a referred older patient (e.g., evaluation of suspected dementia).

5. A home visit otherwise indicated should not be performed if

a. The offer is declined by the patient and/or family;

b. It is inappropriate for the required service (i.e., the assessment/intervention cannot be done to an acceptable standard in the setting of the patient's own home by those performing the home visit);

c. The domicile is inappropriate (e.g., presence of hazards or distractions such as unruly pets, privacy issues);

d. The resources required are excessive (i.e., prolonged travel distance/time, inefficient use of time/resources, opportunity cost);

e. The potential safety of the provider is a significant concern (i.e., safety must be recognized as a priority with a risk assessment done before each home visit).

\section{Program issues:}

Specialized geriatric programs should

1. Plan how home visits will be performed, developing policies and procedures for them;

2. Ensure that the service is integrated within the constantly evolving local system of care for seniors;

3. Support physicians and staff performing home visits (this would include dealing with their training needs);

4. Develop mechanisms to evaluate the quality, effectiveness, and efficiency of the home visiting service;

5. Obtain adequate funding and develop an appropriate infrastructure to support the performance of home visits. 
Technological advances, consumerism, and efforts at cost containment are all driving health care increasingly back to the home. ${ }^{43}$ Some primary care geriatricians have shifted into what has been called a house call practice. ${ }^{44}$ While individual physicians can successfully adopt this type of practice, a specialized geriatric program would be ill advised to move entirely this way - as ill advised as retreating to the borders of the hospital. Rather we must develop an integrated, comprehensive suite of services that includes home visiting to meet the needs of the older population we serve.

\section{CONFLICT OF INTEREST DISCLOSURES}

None declared.

\section{REFERENCES}

1. Thewlis MW. In memoriam: Nascher - Father of Geriatrics. Med Times 1945;73:140-1.

2. Howell T: Editorial: Origins of the British Geriatrics Society. Age Ageing 1974;3:69-72.

3. Anonymous. Eric Barrington Brooke. Lancet 1957;272:221-2.

4. Arcand M, Williamson J. An evaluation of home visiting of patients by physicians in geriatric medicine. $\mathrm{Br}$ Med $J$ 1981;283:718-20.

5. Brooke EB. The place of the out patient department in caring for old people. Med Press 1948;219:400-2.

6. Lord Amulree, Exton-Smith AN, Crockett GS. Proper use of the hospital in treatment of the aged sick. Lancet 1951;i:123-6.

7. Centre for the History of Medicine, BGS Scotland. Domiciliary Visiting by Geriatricians: The Good Old Days? A Witness Seminar in the History of Medicine. Glasgow, UK: Centre for the History of Medicine, Department of Economic and Social History, University of Glasgow; 2007.

8. Fry J, Sandler G. Domiciliary consultations: some facts and questions. BMJ 1988;297:337-8.

9. Lister J. The NHS After 60: For Patients or Profits? London, UK: Middlesex University Press; 2008:117.

10. Taylor IC, McConnell JG. Geriatric medicine: the anatomy of change. Ulster Med J 1994;63:162-9.

11. Anonymous. House calls and home visits. Lancet 1963;i:1409.

12. Meyer GS, Gibbons RV. House calls to the elderly: a vanishing practice among physicians. N Engl J Med 1997;337:1815-20.

13. Hobbs FD. Violence in general practice: a survey of general practitioners' views. BMJ 1991;302:329-32.

14. Mulley GP. Home visiting by consultants. BMJ 1988;296:515-6.

15. Hardy-Thompson C, Orrell MW, Bergmann K. Evaluating a psychogeriatric domiciliary visit service: views of general practitioners. BMJ 1992;304:421-2.

16. Orrell MW, Hardy-Thompson C, Bergmann K. Comparison between general practitioners with high or low use of psychogeriatric domiciliary visit service. Int J Geriatr Psychiatry 1992;7:885-9.

17. Orrell M, Katona C. Do consultant home visits have a future in old age psychiatry? Int J Geriatr Psychiatry 1998;13:355-7.
18. Sutherby K, Srinath S, Strathdee G. The domiciliary consultation service: outdated anachronism or essential part of community psychiatric outreach? Health Trends 1992;24:103-5.

19. Donaldson LJ, Hill PM. The domiciliary consultation service: time to take stock. BMJ 1991;302:449-51.

20. Forsythe M. Domiciliary visits. BMJ 1991,302:426-7.

21. Reardon M, Holmon R, Dewhurst G. A survey of domiciliary consultations in medicine for the elderly. Public Health 1995;109:187-90.

22. Crome $\mathrm{P}$, Malham $\mathrm{A}, \mathrm{Baker} \mathrm{D}$, et al. Domiciliary visits to the old and the mentally ill: how valuable? J $R$ Soc Med 2000;93:187-90.

23. Simpson S, Beavis D, Dyer J, et al. Should old age psychiatry develop memory clinics? A comparison with domiciliary work. Psychiatr Bull 2004;28:78-82.

24. Dennis M, Furness L, Lindesay J, et al. Assessment of patients with memory problems using a nurse-administered instrument to detect early dementia and dementia subtypes. Int $J$ Geriatr Psychiatry 1998;13:405-9.

25. Seymour J, Saunders P, Wattis JP, et al. Evaluation of early dementia by a trained nurse. Int J Geriatr Psychiatry 1994;9:37-42.

26. Galvin JE, Roe CM, Powlishta KK, et al. The AD8: a brief informant interview to detect dementia. Neurology 2005;65:559-64.

27. Trapp-Moen B, Tyrey M, Cook G, et al. In-home assessment of dementia by nurses: experience using the CERAD evaluations. Gerontologist 2001;41:406-9.

28. Page S, Hope K, Bee $\mathrm{P}$, et al. Nurses making a diagnosis of dementia: a potential change in practice? Int J Geriatr Psychiatry 2008;23:27-33.

29. Ramsdell JW, Jackson JE, Guy HJB, et al. Comparison of clinicbased home assessment to a home visit in demented elderly patients. Alzheimer Dis Assoc Disord 2004;18:145-53.

30. Ramsdell JW, Swart JA, Jackson JE, et al. The yield of a home visit in the assessment of geriatric patients. J Am Geriatr Soc 1989;37:17-24.

31. Shievitz AL, Tudiver F, Araujo A, et al. Do elderly people score better on cognitive tests at home? Can Fam Physician 1998;44:1652-6.

32. Ward HW, Ramsdell JW, Jackson JE, et al. Cognitive function testing in comprehensive geriatric assessment: a comparison of cognitive test performance in residential and clinic settings. J Am Geriatr Soc 1990;38:1088-92.

33. Di Monaco M, Vallero F, De Toma E, et al. A single home visit by an occupational therapist reduces the risk of falling after hip fracture in elderly women: a quasi-randomized controlled trial. $J$ Rehabil Med 2008;40:446-50.

34. Cumming RG, Thomas M, Szonyi G, et al. Home visits by an occupational therapist for assessment and modification of environmental hazards: a randomized trial of falls prevention. J Am Geriatr Soc 1999;47:1397-402.

35. Gillespie LD, Robertson MC, Gillespie WJ, et al. Interventions for preventing falls in older people living in the community. Cochrane Database Syst Rev 2009;(2):CD007146. 
36. Jackson JE, Ramsdell JW, Renvall M, et al. Reliability of drug histories in a specialized geriatric outpatient clinic. J Gen Intern Med 1989;4:39-43.

37. Yang JC, Tomlinson G, Naglie G. Medication lists for elderly patients: clinic-derived versus in-home inspection and interview. J Gen Intern Med 2001;16:112-5.

38. Clarfield AM, Bergman H. Medical home care services for the housebound elderly. CMAJ 1991;144:40-5.

39. Tandeter H, Peleg R, Menahem S, et al. Teaching geriatric assessment in home visits: the family physician/geriatrician attachment. Teach Learn Med 2003;15:123-6.

40. Mitsis EM, Jacobs D, Luo X, et al. Evaluating cognition in an elderly cohort via telephone assessment. Int J Geriatr Psychiatry 2010;25:531-9.

41. Gaikwad R, Warren J. The role of home-based information and communications technology interventions in chronic disease management: a systematic literature review. Health Informatics $J$ 2009;15:122-46.

42. American Geriatrics Society Clinical Practice Committee. The Role of House Calls in Geriatric Practice. New York: The Society; 2006.

43. Landers $\mathrm{SH}$. Why health care is going home. $N$ Engl $J$ Med 2010;363:1690-1.

44. Kao H, Conant R, Soriano T, et al. The past, present, and future of house calls. Clin Geriatr Med 2009;25:19-34, v.

Correspondence to: David B. Hogan, MD, FRCPC, FACP, HSC-3330 Hospital Dr. NW, Calgary, AB T2N 4N1. E-mail: dhogan@ucalgary.ca 